\title{
Compassionate Care: Who Provides Care for the Care Giver?
}

\section{Mary Bemker ${ }^{*}$}

School of Nursing, College of Health and Human Services, Touro University Nevada, 874 American Pacific Drive, Henderson, NV 89014, USA

\section{Introduction}

Sandra Ramirez, is an ED nurse with five years experience, dreads going to work. She feels drained, and she does not look forward to interacting with her peers or addressing health concerns of patients. She has to deal with intense trauma throughout each shift. Recently her Primary Care Provider has prescribed sleeping and anxiety medication to help her cope with job demands.

John Jackson, is a Hospice nurse that has been in the field 7 years. Colleagues have noted that he is irritable, complains of difficulty remaining asleep, and continually speaks about his work "bleeding" into his personal life. He often states that he would be better off being a store greeter, and his fellow nurses are beginning to agree with him. They do not appreciate the jokes he shares that focus on death and dying.

Janice Sparks, has worked as a nursing instructor for 20 years. Janice has a history of supporting students who have difficulty passing clinical courses, and she often finds herself with 10 clinical students with major needs. Many of the students look to Janice to help them solve both clinical and life problems. Janice does not want to turn the students away, yet she feels she does not have any more to give. She is becoming cynical as to student's progress and their potential to be good nurses. Janice believes it may be time to retire.

In all of the above cases, one may assume that the nurses are simply tired and need a rest. However, there is a pattern to their behavior that leads one to believe they are suffering from something greater than being tired or overwrought. The combined information lends one to think these instances reflect compassion fatigue.

\section{What is Compassion Fatigue, and Why is It so Prevalent in Nursing?}

Nursing is a profession that provides care to many. We interact in a caring way with clients, patients, family members and others who are suffering. Naturally empathetic, nurses as a whole engage in activities where we are continually exposed to the pain of others. This role is reinforced by societal and cultural expectations of nurses $[1,2,3,4]$.

First identified by Carol Joinson [5], compassion fatigue was recognized among nurses in the Emergency Room. Nurses were worn down by the continued exposure to those in great pain, and some of these nurses had lost their "ability to nurture".This dynamic is a cumulative process, and Compassion Fatigue is progressive if untreated [6].

This conceptualization was expanded upon by Charles Figley [7] when he deemed Compassion Fatigue as a secondary post-traumatic stress disorder. Similar to Post Traumatic Stress Disorder, Figley noted caregivers are impacted by the stress of others rather than experiencing the stress first hand. Whether one significant traumatic event or continual exposure to a series of traumatic events occurs, changes in one's personality results. By looking at these proceedings, one can see that compassion and empathy felt for others creates a dynamic that can result into compassion fatigue.

\section{Publication History:}

Received: June 09, 2017

Accepted: July 18, 2017

Published: July 20, 2017

\section{Keywords:}

Oncological diasease, Oncology nurses, Patients, Consultation

Compassion Fatigue occurs in stages. Compassion discomfort is the hallmark of the first stage. It is here where the nurse may feel a temporary discomfort that may manifest as weariness, lack of enthusiasm and being distracted. This can usually be rectified with separation from work and increased rest [8].

If the situation is not addressed, compassion stress occurs. It is here where the nurse's stress level elevates, and their tolerance and endurance levels decrease. Irritability, difficulty concentrating and decreased performance are identifying variables at this stage [8].

If left uncared for, compassion fatigue is the result. It is in this third phase where nurses are no longer able to care for others. Apathy, poor judgement and the desire to leave their employment may be a few of the dynamics noted. The time frame in which these dynamics progress is individual in nature. When one reaches the stage where compassion fatigue is noted a variety of indicators are seen.

\section{Signs and Symptoms}

Signs and symptoms of compassion fatigue are multifaceted, and the final result is often compounded by the impact of these dynamics reinforcing the negative expressions of compassion fatigue. Physical symptoms may be difficult to directly link to compassion fatigue alone. Complaints such as a headache or gut-wrenching distress by themselves will not indicate compassion fatigue [9]. Therefore, it is important to address symptomatology as a pattern of outcomes that reflect this Secondary Stress Disorder.

Physiological symptoms of Compassion Fatigue can include being nervous or generally upset. One's pattern of sleep may be affected, and results of such can range anywhere from insomnia to excessive sleeping. Psychological factors may be noted as irritation or being extremely impatient with self, others and situations at large. Judgement and recall can also be negatively impacted [9]. Overall disinterest or malaise could be noted, with distancing from others or social events resulting [10]. In addition, separating oneself from spiritual supports often go along with these other dynamics [10].

"Corresponding Author: Dr. Mary Bemker, School of Nursing, College of Health and Human Services, Touro University Nevada, 874 American Pacific Drive, Henderson, NV 89014, USA; E-mail: mary.bemker@tun.touro.edu

Citation: Bemker M (2017) Compassionate Care: Who Provides Care for the Care Giver?. Int J Nurs Clin Pract 4: 244. doi: https://doi.org/10.15344/2394$4978 / 2017 / 244$

Copyright: @ 2017 Bemker. This is an open-access article distributed under the terms of the Creative Commons Attribution License, which permits unrestricted use, distribution, and reproduction in any medium, provided the original autho and source are credited. 
In turn, the consequences of these symptoms create a downward spiral that can be self-perpetuating. The result can be disinterest in the current employment or nursing in general. Ethical considerations might be compromised [11], and job performance could be diminished [10].

\section{Burnout}

Often confused with Compassion Fatigue, burnout is a condition evidenced by chronic stress. This condition results in physical and emotional exhaustion, detachment and belief that one is ineffective. Incivility, blurring of job requirements, continued unscheduled overtime, and lack of support are but a few of the dynamics that perpetuate burnout. Burnout has a slower manifestation than compassion fatigue, and the key phenomena are linkedto occupational issues rather than the connection to specific individuals and their pain $[12,13]$.

Common signs of burnout include cynicism, lack of motivation, lethargy, low productivity, and disenchantment with one's place of work. Abuse of chemical substances (use of illegal substances or misuse of legal and prescribed substances), changes in eating and sleeping patterns, and generalized symptoms of stress (e.g., headache, backache) are often noted [12].

(For a free screening tool for Compassion Fatigue and burnout, go to the Compassion Fatigue Project at: http://www.compassionfatigue. org/pages/selftest.html.)

\section{Burnout}

Compassion Satisfaction is the positive outcomes experienced by a nurse when helping others and creating a positive change in the world. It allows an individual to determine the degree in which they feel successful as nurses, and it also determines/is determined the level the individual believes they are supported by their colleagues $[14,15]$

Having compassion fatigue does not rule out the feeling of compassion satisfaction or vice versa. It has been shown that Compassion Fatigue and Compassion Satisfaction are inversely related [16].Thus, increasing Compassion Satisfaction minimizes the potential for Compassion Fatigue. Just the fact that one is aware of such, provides the potential for self-help in relation to such.

\section{Proactive Interventions}

In addition to recognition of Compassion Fatigue, personal and situational dynamics of Compassion Fatigue, and the need to enhance Compassion Satisfaction, the nurse needs to find ways to increase work - life balance. Making the employment experience a part of one's life instead of the life focus can do much to minimize the impact of secondary stress.

This balance can be accomplished in a variety of ways. Having interests outside of work is key, and self-care is paramount. Setting up "dates" with others to be socially active, is one way that a nurse can insert activity outside of work into their lives. (Having a committed event also decrease the potential that one will come up with reasons not to follow through on connecting with others.) When a nurse chooses this type of intervention, it is important to do something that is fun, rather than something that feels like a requirement or work.
If a nurse regenerates more when alone, reading, painting and other creative outlets can support self-care. Meditation, yoga, self-reflection and journaling can also be a positive means to get in touch with inner peace. Whatever one chooses, it is important that the activity enhances the nurse's life, and it is pleasurable to them.

Group support can be a proactive measure for nurses. Having a journal club with articles addressing self-care, compassion fatigue, burnout and other related topics can provide education on the topic while offering an understanding that they are not alone with the feelings and experiences that are occurring. Being a part of a work support group, group counseling for health professionals, or any other group experience directed toward a safe outlet for feelings and mutual support system can provide perspective and understanding of the dynamics that are in play.

Employee Assistance Programs can also be a valuable resource for the nurse. Many times, one may not be aware of the underlying mechanics that are precipitating feelings and outcomes. Nurses do not have to be "Wonder Woman" or "Wonder Man" to be a success. Having someone to speak with about such feelings, in safe and confidential setting, may provide the insights on how to deal with work issues and work-life balance.

Knowing how to disconnect and relax can be a challenge for many. Promoting or using a relaxation center at work, meditating and taking mindfulness classes, exercising and being physically active, contemplation, and other relaxation activities (e.g., getting a massage or receiving a Reiki treatment) can serve to provide means to combat stress, Compassion Fatigue and burnout [17].

\section{Conclusion}

Nursing by its very definition is a caring profession. Knowing how to care for self when faced with stressors that are associated with Compassion Fatigue offers a means to support resiliency. With "sicker patients" noted in healthcare environments, it is more important than ever for nurses to care for self. There are some strategies offered here that might support reduction in the potential for Compassion Fatigue. Feel free to use these and to come up with some other options on your own. Nurses are great caregivers, and it is important that self is included in those receiving care.

\section{Competing Interests}

The authors declare that she has no competing interests.

\section{References}

1. Carroll SM, Rosa KC (2016) Role and image of nursing in children's literature: A qualitative media Analysis. J Pediatr Nurs 31: 141-151.

2. Chambers C, Ryder E (2016) Compassion and caring in nursing, CRC Press.

3. Girvin J, Jackson D, Hutchinson M (2016) Contemporary public perceptions of nursing: a systematic review and narrative synthesis of the international research evidence. J Nurs Manag 24: 994-1006.

4. Rhodes M, Morris A, Lazenby R (2011) Nursing at its best: Competent and caring. Online $\mathrm{J}$ Issues Nurs 16: 10.

5. Joinson C (1992) Coping with Compassion Fatigue. Nursing 116: 118-119.

6. Cole J (2011) Compassion fatigue: If the nurse's ability to care is affected, quality of care could suffer. Advance Healthcare Networks.

7. Figley C (1995) Compassion fatigue: Coping with secondary traumatic stress disorder in those who treat the traumatized. New York, NY: BrunnerRoutledge. 
Citation: Bemker M (2017) Compassionate Care: Who Provides Care for the Care Giver?. Int J Nurs Clin Pract 4: 244. doi: https://doi.org/10.15344/23944978/2017/244

8. Coetzee $\mathrm{S}$, Klopper $\mathrm{H}$ (2010) Compassion fatigue within nursing practice: a concept analysis. Nurs Health Sci 12: 235-243.

9. Lombardo B, Eyre C (2011) Compassion fatigue: A nurse's primer. Online $\mathrm{J}$ Issues Nurs 16:3.

10. Boyle DA (2011) Countering compassion fatigue: A requisite nursing agenda. Online J Issues Nurs 16: 2

11. Lachman VD (2016) Ethics, law, and policy. Compassion fatigue as a threat to ethical practice: Identification, personal and workplace prevention/ management strategies. MedSurg Nursing 25: 275-278.

12. Heidi B (2013) Preventing and managing compassion fatigue and burnout in Nursing. ESSAI 11: 11

13. Portnoy D (2011). Burnout and compassion fatigue: Watch for the signs Health Progress.

14. Conrad d, Kellar-Guenther $Y$ (2006) Compassion fatigue, burnout, and compassion satisfaction among Colorado child protection workers. Child Abuse Negl 30: 1071 -1080.

15. Stamm BH (2002) Measuring compassion satisfaction as well as fatigue: Developmental history of the compassion satisfactionand fatigue test. In Figley CR (Ed.), Treating Compassion Fatigue (pp. 107-119). New York: Brunner-Routledge.

16. Yoder E (2010) Compassion fatigue in nurses. Applied Nursing Research 23:191-197.

17. Job burnout: How to spot it and take action (2017). 